\title{
Neues über die elektrophile Aromatensubstitution
}

Die elektrophile Aromatensubstitution gehört zu den wichtigsten Reaktionen in der organischen Chemie; unzählige Synthesen aromatischer Verbindungen beginnen mit der elektrophilen Einführung eines Substituenten in den aromatischen Ring (Halogenierung, Nitrierung, Sulfonierung, Alkylierung, Acetylierung u.a.).

\section{Der o-Komplex-Mechanismus}

Im Gegensatz zur nucleophilen Aromatensubstitution, für die mehrere mechanistische Alternativen gesichert sind (bimolekularer Austausch, Arin-Mechanismus, Dissoziations-Mechanismus), verlaufen fast alle bekannten elektrophilen Aromatensubstitutionen nach dem $\sigma$-Komplex-Mechanismus über Cyclohexadienylkationen (Abbildung 1) [1].

Aus Untersuchungen des kinetischen Isotopeneffekts (Vergleich der Reaktionsgeschwindigkeiten von Wasserstoff- und Deuteriumverbindungen) konnte für die meisten Reaktionen nachgewiesen werden, daß die Bildung der $\sigma$-Komplexe der langsamste, d. h. geschwindigkeitsbestimmende Schritt ist $\left(\mathrm{k}_{1} \ll \mathrm{k}_{2}\right)$. Der bei der aromatischen Sulfonierung auftretende kleine Isotopeneffekt kommt daher, daß in diesem Fall die $\sigma$-Komplex-Zwischenstufe leicht zu den Ausgangsstoffen zurückreagiert $\left(k_{-1} \sim k_{2}\right)$.

Als weitere Reaktionszwischenstufen bei der elektrophilen Aromatensubstitution werden $\pi$-Komplexe diskutiert. In vielen Fällen lassen sich Additionskomplexe (engl.: encounter pairs), die durch die Wechselwirkung des aromatischen Systems mit dem meistens positiv geladenen Elektrophil entstehen können, ohne Schwierigkeiten nachweisen. Allerdings bestehen kontroverse Ansichten darüber, ob diese $\pi$-Komplexe notwendige Zwischenstufen bei der elektrophilen Aromatensubstitution sind und in welcher Weise sie in die o-Komplexe übergehen.

$\sigma$-Komplexe konnten ${ }^{1} \mathrm{H}-\mathrm{NMR}$-spektroskopisch eindeutig nachgewiesen werden, ihre Isolierung und Charakterisierung gelang in jüngster Zeit bei Umsetzungen symmetrischer Triaminobenzole mit Elektrophilen (Abbildung 2a) [2]. Diese durch drei Aminogruppen stark mesomeriestabilisierten $\mathrm{Zwi-}$ schenverbindungen stellen die positiv geladenen Analoga zu den ebenfalls mesomeriestabilisierten, jedoch negativ geladenen Meisenheimer-Komplexen bei der nucleophilen Aromatensubstitution dar (Abbildung 2b)

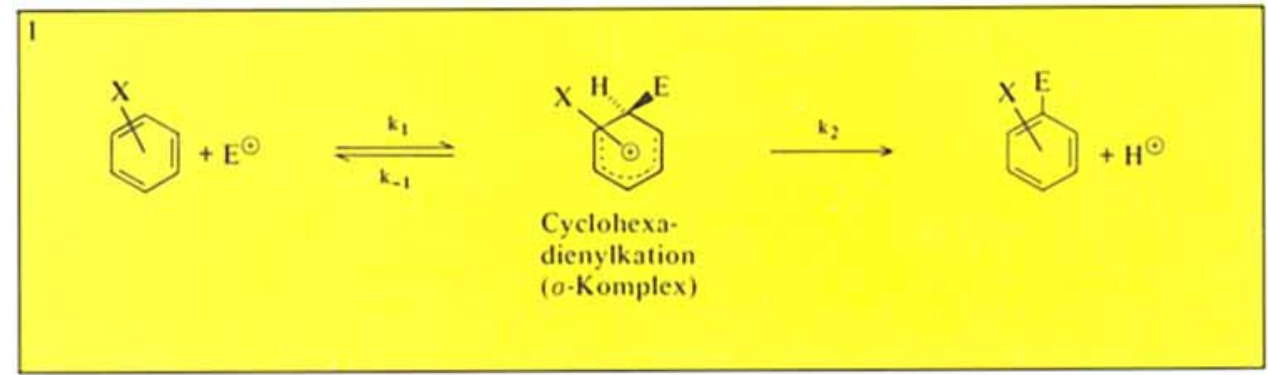

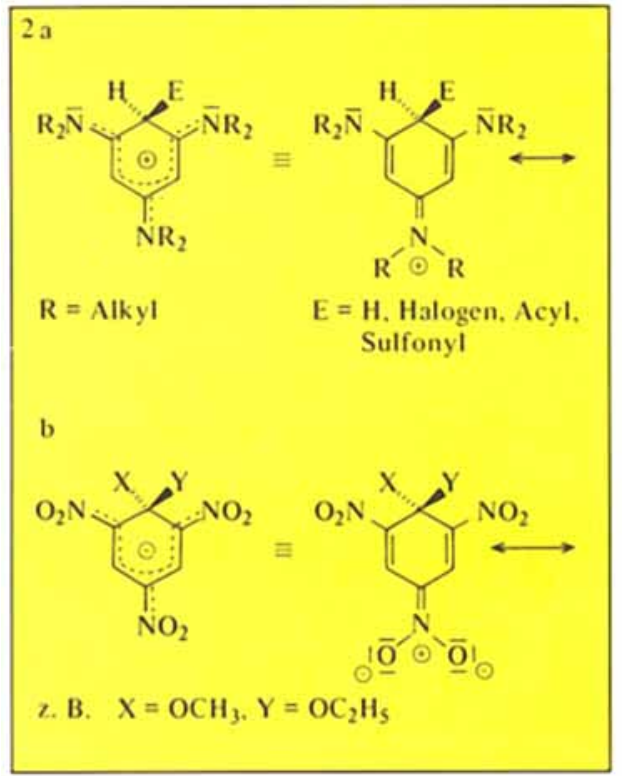

und erlauben - wie diese - Untersuchungen der beiden Teilschritte der Reaktionen.

Das Auftreten kationischer Zwischenstufen und ihre grundlegende Bedeutung bei der elektrophilen Aromatensubstitution ist schon 1928 erkannt worden. Die quantenmechanische Behandlung der Orientierung bei der Zweitsubstitution durch Wheland im Jahre 1942 führte zu dem Ergebnis, daß die $\sigma$ Komplexe als Modell für den Úbergangszustand der elektrophilen A romatensubstitution gut geeignet sind. Gerade diese theoretische Fundierung der alten Orientierungsregeln, die es nun erlaubte, durch Berechnung oder Abschätzung der Stabilität der kationischen Zwischenstufen Voraussagen über Reaktivität und Orientierung bei elektrophilen Aromatensubstitutionen zu machen, war so faszinierend, $d a ß$ andere denkbare Reaktionsmechanismen völlig vernachlässigt wurden. Auch in der Lehre wird diese Reaktion als Musterbeispiel für quantitativ voraussagbare Reaktionsabläufe dargestellt.

\section{Abweichungen vom $\sigma$-Komplex- Mechanismus}

Experimentell wurden schon immer ge-
Abb. 1. $\sigma$-Komplex-Mechanismus der elektrophilen Aromatensubstitution. Erster Schritt: Reaktion eines Elektrophils $E^{\oplus}$, das als Kation, als positives Ende eines Dipols oder als induzierter Dipol mit dem nucleophilen Aromaten zu einer kationischen $Z$ wischenverbindung ( $\sigma$-Komplex, Arenium-Ion, Wheland-Zwischenverbindung) führt. Zweiter Schritt: Abspaltung des Elektrofugs, in den meisten Fällen eines Protons, unter Ausbildung des substituierten Aromaten.

Abb. 2. a) Isolierbare $\sigma$-Komplexe bei der Umsetzung von Elektrophilen mit 1,3,5Triaminobenzolen. b) Meisenheimer-Komplex, isolierbare Zwischenstufe bei der nucleophilen aromatischen Substitution.

wisse Abweichungen von dieser StrukturReaktivitäts-Beziehung gefunden. In den letzten Jahren sind nun auch mechanistische Alternativen zum $\sigma$-Komplex-Mechanismus entwickelt worden, die vor allem eine Deutung der mit diesem Mechanismus nicht $\mathrm{zu}$ vereinbarenden Produktbildung erlauben. Reaktionen dieses Typs werden meistens als "ungewöhnliche“ (engl:: nonconventional) elektrophile Aromatensubstitutionen [3] bezeichner. Wenn auch bisher mehr die theoretische Erklärung ungewöhnlicher Produktbildungen im Vordergrund dieser Untersuchungen stand, so zeichnet sich doch jetzt schon $a b, d a ß$ die gezielte Anwendung der dabei gewonnenen Erkenntnisse zu einer beachtlichen Ausweitung der synthetischen Möglichkeiten in der Aromatenchemie führen kann.

Nach den heutigen Kenntnissen lassen sich die Reaktionen, bei denen Abweichungen vom üblichen Mechanismus auftreten, in zwei Gruppen einteilen:

Ipso-Reaktionen: Hierbei greift das Elektrophil an einem bereits substituierten CAtom des Ringes an. Meistens ist der Substituent eine schlechtere Abgangsgruppe als 
das Proton, so daß andere Folgereaktionen des $\sigma$-Komplexes gegenüber der Rearomatisierung zum Substitutionsprodukt bevorzugt werden (s. unten).

Radikal-Kation-Bildung: Ist der Zweielektronenübergang vom Aromaten zum Elcktrophil erschwert, so kann es auch zu einem Einelektronenübergang unter Bildung eines Radikalkations kommen, das dann verschiedene Folgereaktionen eingehen kann. Dieser Reaktionsablauf wird vor allem dann beobachtet, wenn der normale elektrophile Angriff sterisch gehindert ist oder wenn schwache Elektrophile mit ausreichendem Oxidationspotential eingesetzt werden (s. unten).

\section{Reversibilität elektrophiler Aromatensub-} stitutionen (ipso-Reaktionen)

Für die Anwendung der Orientierungsregeln bei Mehrfachsubstitutionen ist ein irreversibler Verlauf der Substitution grundlegende Voraussetzung. Es ist schon lange bekannt, daß diese Voraussetzung bei der Alkylierung und der Sulfonierung nicht immer erfüllt ist, da bei diesen Rcaktionen unter energischen Bedingungen Isomerenverteilungen gefunden werden, die nicht den aus den $\sigma-$ Komplex-Stabilitäten abzuleitenden Verteilungen entsprechen. Die Sulfonierung des Naphthalins z. B. führt bei $80^{\circ} \mathrm{C}$ ausschließlich zur 1-Naphthalinsulfonsäure, während bei $160^{\circ} \mathrm{C}$ nur die 2-Naphthalinsulfonsäure entsteht (Abbildung 3).

Ein reversibler Reaktionsverlauf wird meist nur bei reaktiven Aromaten (Alkylaromaten, Naphthalin u.ä.) beobachtet. Neben der Reaktivität, die den Protonierungsschritt (mit der Geschwindigkeitskonstanten $k_{1}$ ) beeinflußt, ist jedoch auch die Austrittstendenz des Elekrofugs $\mathrm{E}^{\oplus}$ sowie die Nucleophilie des Gegenions A entscheidend(Abbildung 4)

Es wurde versucht, die verschiedenen Substituenten bezüglich ihrer elektrofugen Austrittstendenz zu ordnen, wobei zwischen Gruppen, die ohne Unterstützung eines $\mathrm{Nu}$ cleophils als Kation austreten können $\left(S_{N} 1\right.$ Typ), und solchen, die nur mit Hilfe eines Nucleophils abgelöst werden $\left(\mathrm{S}_{\mathrm{N}} 2-T_{y}\right)$, zu unterscheiden ist. Es ergeben sich folgende zwei Reihen mit zunehmender Austrittstendenz [4]:

$\mathrm{S}_{\mathrm{N}} 1-\mathrm{Typ}: \mathrm{NO}_{2}^{\oplus}<\mathrm{CH}_{3}-\stackrel{\oplus}{\mathrm{C} H}-\mathrm{CH}_{3} \sim \mathrm{SO}_{3}$ $<\stackrel{\oplus}{\mathrm{C}}\left(\mathrm{CH}_{3}\right)_{3} \sim \mathrm{ArN}_{2}^{\oplus} \sim \mathrm{ArCHOH}^{\oplus}<\mathrm{CO}_{2}$
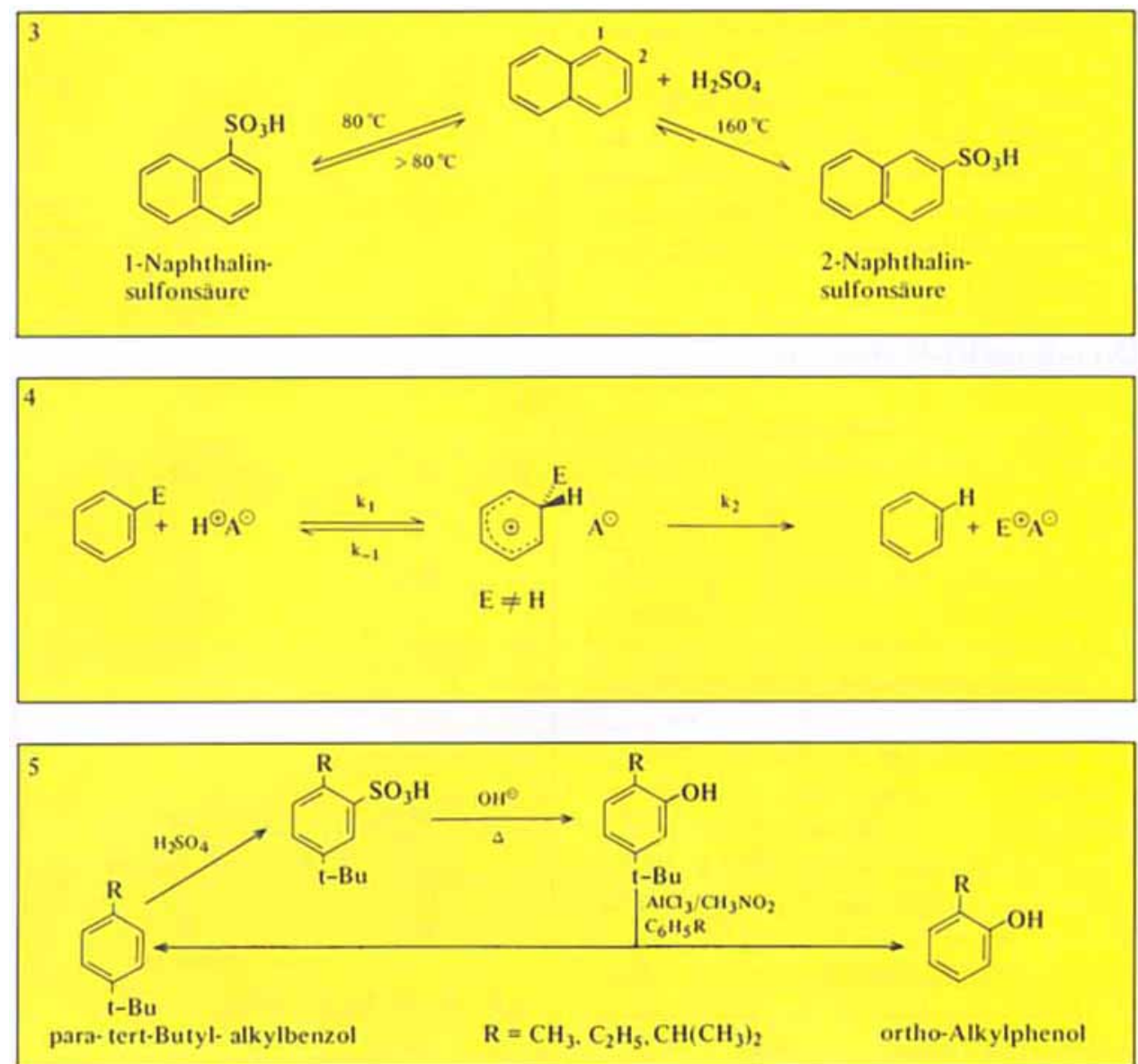

$\mathrm{S}_{\mathrm{N}} 2-$ Typ: $\mathrm{CH}_{3}^{\oplus}<\mathrm{Cl}^{\oplus}<\mathrm{Br}^{\oplus}<\mathrm{D}^{\oplus} \sim \mathrm{RCO}^{\oplus}$ $<\mathrm{H}^{\oplus} \sim \mathrm{J}^{\oplus}<\left(\mathrm{CH}_{3}\right)_{3} \mathrm{Si}^{\oplus} \quad($ Ar $=$ Ary $\left.]\right)$

Die Reihenfolge beim $\mathrm{S}_{\mathrm{N}} 1-\mathrm{T}$ yp entspricht erwartungsgemäß der zunehmenden Kationenstabilisierung. Beim $\mathrm{S}_{\mathrm{N}} 2-\mathrm{Typ}$ ist eine starke Abhängigkeit vom nucleophilen Partner zu erwarten; bei Abwesenheit eines geeigneten Nucleophils kommt es zu Isomerisierungen (Wanderungen des Elektrofugs $\mathrm{E}$ innerhalb des Moleküls). Gerade über den wichtigen Einfluß des Nucleophils auf die Abspaltung von $\mathrm{E}^{\oplus}$ aus den $\sigma$-Komplexen ist noch wenig bekannt.

\section{Beispiele für die präparative Anwendung} der Reversibilität

Präparativ wurde die Reversibilität der elektrophilen Aromatensubstitution hauptsächlich für Isomerisierungs- und Abspaltungsreaktionen bei Alkylaromaten und aromatischen Sulfonsäuren genutzt, in neuerer Zeit jedoch auch bei Halogen- und Acylaromaten.

Anionen-Einfluß auf die Abspaltung von Brom aus Aromaten [5]

Die Abhängigkeit der Abspaltung eines $\mathrm{Hal}^{\oplus}$
(Elektrofug vom $\mathrm{S}_{\mathrm{V}}$ 2-Typ) von der Nucleophilie des Anions $\left(\mathrm{Br}^{\ominus}>\mathrm{Cl}^{\ominus} \gg \mathrm{ClO}_{4}^{\ominus}\right)$ läßt sich experimentell in schöner Weise beim $\mathrm{Er}$ hitzen verschiedener Salze des 2-Brom-dimethylanilins in Chloroform auf $120^{\circ} \mathrm{C}$ zeigen (Abbildung 6).

Die konkurrierende Protonierung am Stickstoff und die im geschlossenen System ablaufenden Folgereaktionen des Dimethylanilins mit dem abgespaltenen $\mathrm{ABr}$ führen zu einem Produktgemisch, das mit den angeführten Ergebnissen in Einklang steht.

\section{tert-Butylreste als Schutzgruppen [6]}

Ein Beispiel für die präparative Anwendung einer reversiblen Substitutionsreaktion in Gegenwart eines tert-Butylrestes als Schutzgruppe ist in Abbildung 5 erläutert.

\section{Reversibilität der Aromatenacylierung [7]}

Die Nichtbeachtung der Reversibilität ist bei der Acylierung besonders gravierend. So wird z.B. die fast ausschließliche $(>95 \%)$ Bildung des para-Isomeren bei der Acetylierung von Toluol mit Acetylchlorid/ $\mathrm{AlCl}_{3}$ durch die sterische Hinderung der ortho- 


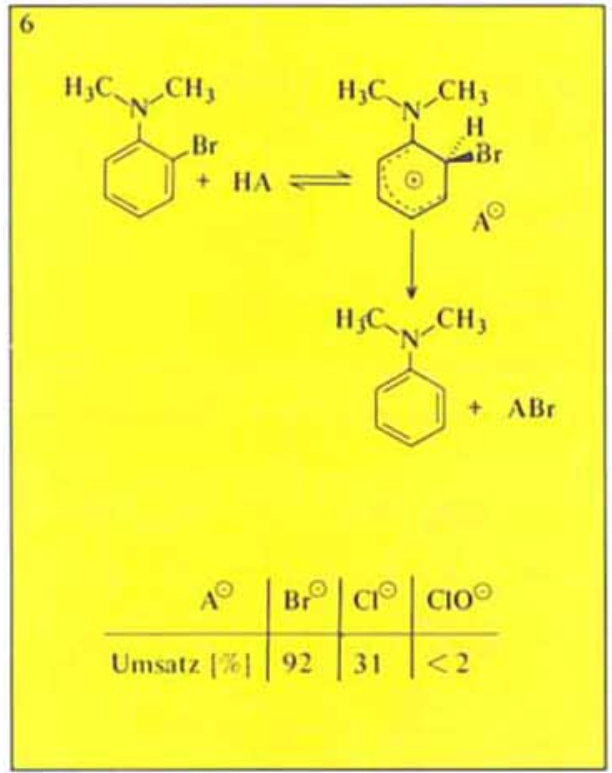

Abb. 3. Sulfonierung von Naphthalin. Die durch bessere Mesomeriestabilisierung des $\sigma$-Komplexes bedingte Begünstigung der Sulfonierung in Position 1 (kinetische Kontrolle) ist bei erhöhter Temperatur reversibel. Bei erhöhter Temperatur wird dann zwar die Aktivierungsschwelle zur Bildung der 2-Naph thalinsulfonsäure überwunden, nicht jedoch die für die Rückreaktion, so daß das thermodynamisch stabilere 2-Isomere entsteht.

Abb. 4. Reversibilität der elektrophilen Aromatensubstitution.

Abb. 5. Selektive Herstellung von orthoAlkylphenolen. Alkylbenzole mit tertButylgruppen in para-Stellung können in ortho-Position sulfoniert und anschließend zu den entsprechenden Phenolen umgesetzt werden. Mit $\mathrm{AlCl}_{3} / \mathrm{CH}_{3} \mathrm{NO}_{2}$ als mildem Friedel-Crafts-Katalysator gelingt dann die selektive Ubertragung der tertButyl-Schutzgruppe auf die Ausgangsalkylbenzole (Kreisprozeß).

Abb. 6. Einfluß des Anions $\mathrm{A}^{\ominus}$ auf die $\mathrm{Br}^{\oplus}-$ Abspaltung. Der angegebene Umsatz ist nach drei Stunden erreicht.

Abb. 7. Bildung und mögliche Folgereaktionen von ipso- $\sigma$-Komplexen.

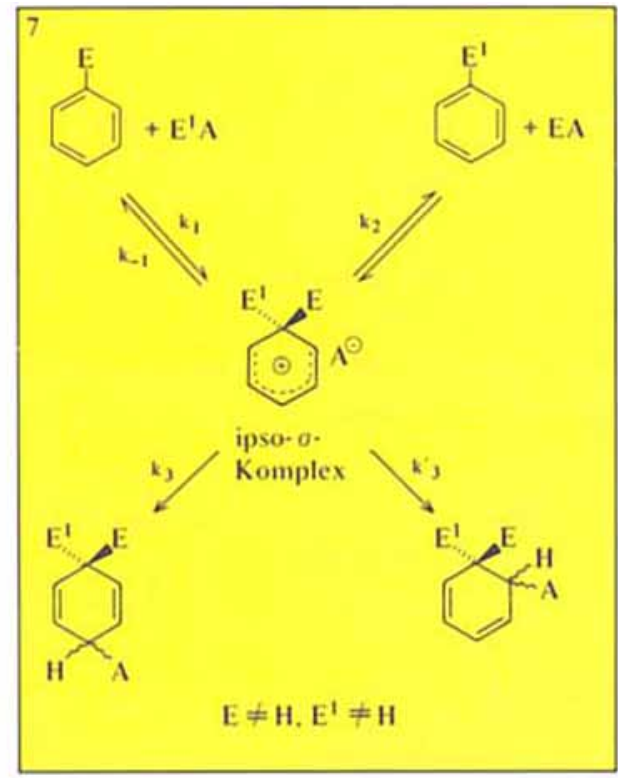

Reaktion durch das stark raumerfüllende Acylierungsagens gedeutet. Neuere Arbeiten haben jedoch gezeigt, daß das ortho-Isomere unter den Reaktionsbedingungen praktisch quantitativ zum p-Acetyltoluol umlagert.

Die Bildung der $\sigma$-Komplexe ist bei orthosubstituierten Acylaromaten besonders begünstigt, da die im planaren Aromaten vorhandene sterische Hinderung der beiden nachbarständigen Gruppen im $\sigma$-Komplex weitgehend aufgehoben ist. Für das paraIsomere fällt diese sterische Begünstigung der $\sigma$-Komplexbildung weg.

Ein interessantes Beispiel für den sterischen Einfluß auf die Reversibilität der Aromatenacylierung ist die quantitative Retro-FriesUmlagerung des 2-Benzoyl-3,5-dimethylphenols zum entsprechenden Phenolester 1 [8]. Die Abspaltung der Benzoylgruppe,

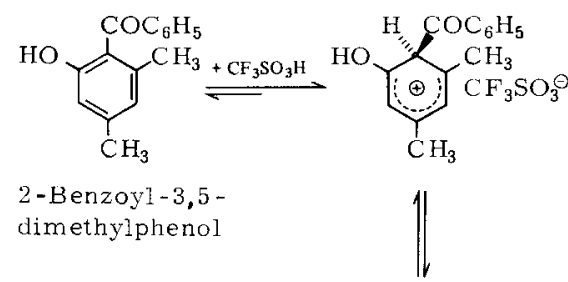

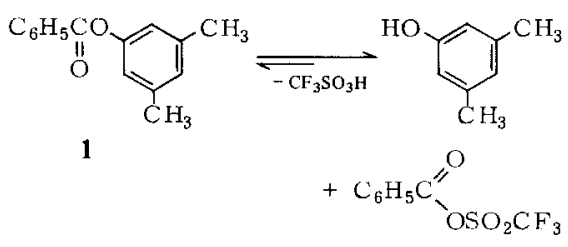

$-\mathrm{CO}-\mathrm{C}_{6} \mathrm{H}_{5}$, vom Ring ist in allen Positionen sterisch begünstigt.

\section{Elektrophile Addition an Aromaten}

Greift beim ipso-Angriff nicht wie bei der Umkehrung der normalen Substitution das Proton, sondern ein anderes Elektrophil an, so entstehen ipso- $\sigma$-Komplexe, die neben der erschwerten Rearomatisierung vor allem Additionsreaktionen eingehen (Abbildung 7). Die Frage, ob sich der Substituent $E$ unter Bildung des ipso-Substitutionsproduktes abspaltet oder ob sich das Gegenion $A^{\ominus}$ addiert, hängt sowohl von der Austrittstendenz von $E$ und $E^{1}$ als auch der Nucleophilievon $A^{\ominus} a b$.

Ausführlich wurde unter diesem Gesichtspunkt bisher lediglich die Nitrierung von Alkylaromaten untersucht [9]; in viel geringerem Maße noch die Halogenierung. Den Einfluß der Austrittstendenz des Elektrofugs $\left(E^{\oplus}\right.$ oder $\left.E^{1 \oplus}\right)$ auf die Produktbildung zeigt ein Vergleich der Nitrierung von Isopropylund Methyl-Aromaten (Abbildung 8).

Daß sich die Produktbildung nicht nur durch die Abspaltungstendenz der Substituenten, sondern auch durch Variation der Reaktionsbedingungen entscheidend beeinflussen läßt, zeigt das nachstehende Beispiel der Nitrierung von Hemellitol mit einem Gemisch aus Salpetersäure und Essigsäureanhydrid.

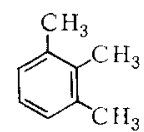<smiles>CC(=O)Oc1cc(C)c(C)c(C)c1</smiles>

Hemellitol

5 -Acetoxyhemellito

$-5{ }^{\circ} \mathrm{C} \downarrow \mathrm{HNO}_{3} / \mathrm{CH}_{3} \mathrm{CO}_{2} \mathrm{O}$ $\uparrow-\mathrm{HNO}_{2}$

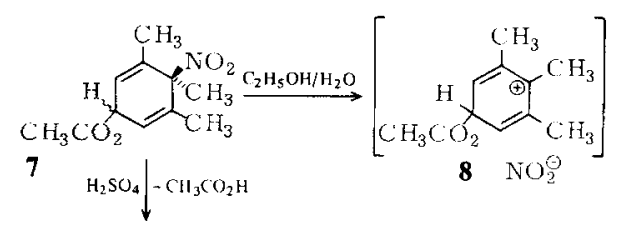<smiles>Cc1cccc(C)c1[N+](=O)[O-]</smiles><smiles>Cc1cccc(C)c1C</smiles><smiles>Cc1ccc([N+](=O)[O-])c(C)c1C</smiles><smiles>Cc1cccc([N+](=O)[O-])c1C</smiles>

4 -vitrohemellitul 
Das Additionsprodukt 7 entsteht unter den angegebenen Reaktionsbedingungen $\mathrm{zu}$ ca. $40 \%$ neben den normalen Substitutionsprodukten. Unter neutralen Solvolysebedingungen $\left(\mathrm{C}_{2} \mathrm{H}_{5} \mathrm{OH} / \mathrm{H}_{2} \mathrm{O}\right)$ entsteht aus 7 durch Nitritabspaltung 8 , das quantitativ in das 5-Acetoxyhemellitol übergeht, während im stark sauren Medium (75-proz. $\mathrm{H}_{2} \mathrm{SO}_{4}$ ) die Abspaltung des Acetations zu 9 begünstigt ist, aus dem durch zweimalige Wanderung der $\mathrm{NO}_{2}$-Gruppe jeweils an das benachbarte C-Atom (1,2-Wanderung) und Deprotonierung ebenfalls quantitativ 4-Nitrohemellitol entsteht.

Diese Befunde zeigen, daß von den beiden isomeren Mononitro-hemellitolen 5-Nitrohemellitol nur über die elektrophile Substitution entstehen kann, während 4-Nitrohemellitol unter geeigneten Reaktionsbedingungen auch über einen Additions-Umlagerungs-Mechanismus gebildet wird. Unter Berücksichtigung dieser Befunde kann das Isomerenverhältnis durch Wahl der Reaktionsbedingungen gezielt verändert werden.

\section{Reaktionen in der Seitenkette von} Alkylaromaten [10]

Dic bekannte Wasserstoff-Substitution in Alkylseitenketten von Aromaten wird im allgemeinen als radikalische Substitution beschrieben und eignet sich besonders gut zur Einführung von Halogenatomen. Eine Anderung der Reaktionsbedingungen kann aber auch hier zu einem andersartigen Reaktionsablauf und damit zu einer anderen Produktbildung führen. Die Halogenierung von Alkylaromaten ist ein bekanntes Lehrbuchbeispiel dafür: Unter den Bedingungen einer Radikalreaktion (Sonne, Siedehitze) wird die Seitenkette halogeniert (SSS-Regel), während die Begünstigung einer elektrophilen Reaktion (Katalysator, Kälte) ausschließlich zur Kernsubstitution führt (KKK-Regel). In jüngster Zeit wurde bei Nitrierungen unter elektrophilen Bedingungen jedoch auch Seitenkettensubstitution beobachtet: So führt die Nitrierung von Pentamethylbenzol zu einem Gemisch von Produkten, die im Kern (10) oder in der Seitenkette (11) substituiert sind.

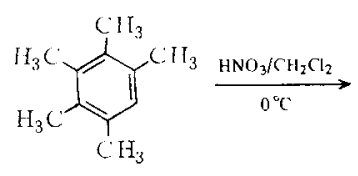

Pentamethy

benzol
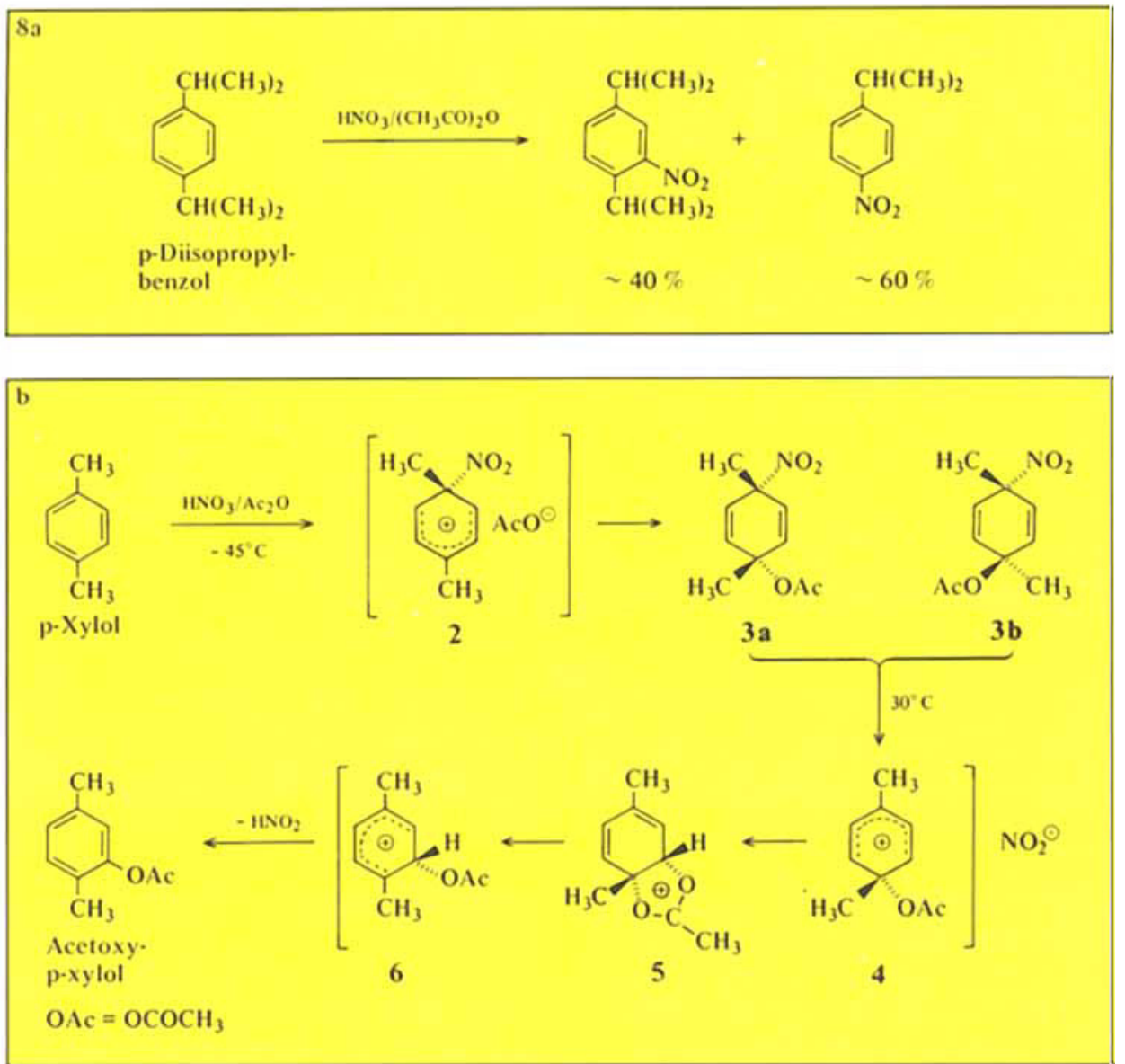<smiles>Cc1c(C)c(C)c([N+](=O)[O-])c(C)c1C</smiles>

Umfangrciche Untersuchungen über den Einfluß von Substituenten und Reaktionsbedingungen auf die Produktbildung führten zu der Schlußfolgerung, daß auch diese Reaktionen durch einen ipso-Angriff des Elektrophils eingeleitet werden und der dabei gebildete $\sigma$-Komplex auf andere Weise weiterreagiert. So können, wie das Beispiel unten zeigt, bei der Nitrierung von Hexamethylbenzol nach der Deprotonierung des ipso- $\sigma$ Komplexes 12 die isomeren Cyclohexadicnmethide 13 und 14 entstehen und unter Austritt des Nitrit-Ions das mesomeriestabilisierte Benzylkation 15 bilden. Durch Anlagerung der im Reaktionsgemisch vorhandenen Nucleophilen $\mathrm{NO}_{2}^{\ominus}$ oder $\mathrm{NO}_{3}{ }^{\ominus}$ entstehen dann die in der Seitenkette substituierten Benzolderivate 16 und 17. $17 \mathrm{kann}$ nicht nur über eine Addition von $\mathrm{NO}_{2}{ }^{\ominus}$ an das Benzylkation 15, sondern auch über die Nitrierung der olefinischen $\mathrm{CH}_{2}$-Gruppe in 13 und anschließende Rearomatisierung unter Austritt von $\mathrm{NO}_{2}^{\oplus}$ entstehen.
Abb. 8. a) Ipso-Substitution bei der Nitrierung von p-Diisopropylbenzol. Wegen der guten elektrofugen Austrittstendenz des Isopropylkations entsteht bei dieser Reaktion mehr ipso-Substitutionsprodukt als normales Nitrierungsprodukt. b) Elektrophile Addition bei der Nitrierung von pXylol. Neben dem Nitro-p-xylol entsteht bei der Nitrierung unter den angefuihrten Bedingungen über den Komplex 2 ein Gemisch der Stereoisomeren $3 \mathrm{a}$ und $3 \mathrm{~b}$, die bei höherer Temperatur über die $\sigma$-Komplexe 4 und 6 zum Acetoxy-p-xylol reagieren. Dafür ist sicher die begünstigte Bildung von 5 mit entscheidend. 

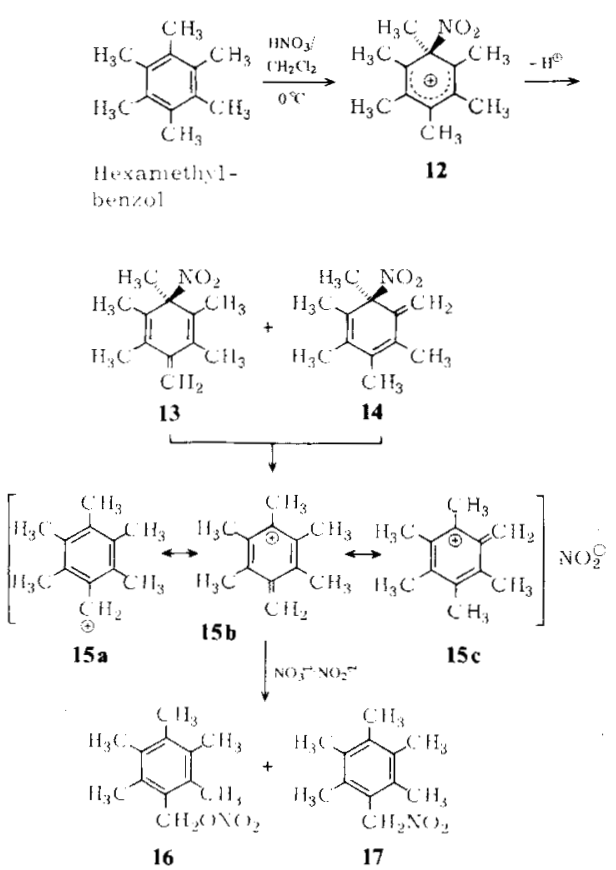

Die Nitrierung von Pentamethylbenzol mit dem Nitroniumsalz $\mathrm{NO}_{2}{ }^{\oplus} \mathrm{PF}_{6}{ }^{\ominus}$ in Acetonitril zeigt, daß auch andere Nucleophile an die im Verlauf der Reaktion gebildeten Benzylkationen addiert werden können. Als Hauptprodukte erhält man Pentamethylnitrobenzol (20\% Ausbeute), das durch normale Kernsubstitution entsteht, sowie das acetylierte Benzylamin 19 (38\% Ausbeute). Die Bildung von 19 kann wie oben über einen ipso-Angriff des $\mathrm{NO}_{2}{ }^{\oplus}$ und die Addition von Acetonitril als Nucleophil an ein Benzylkation gedeutet werden. Das als Zwischenprodukt entstehende Iminocarbeniumion 18 reagiert dann mit Wasser zu 19 weiter.

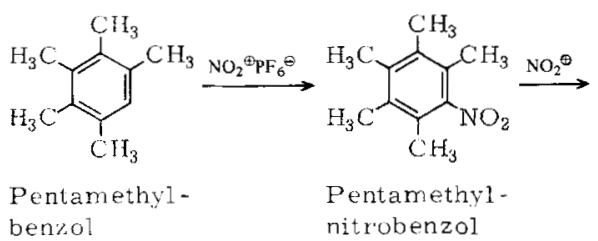<smiles>Cc1c(C)c(C)c([N+](=O)[O-])c(C(C)C)c1C</smiles><smiles>CC#CCN(CC)Cc1c(C)c(C)c(CNC(C)=O)c(C)c1[N+](=O)[O-]</smiles>

18
Häufig gehen den Seitenkettensubstitutionen Additionsreaktionen voraus. Durch Variation der Reaktionsbedingungen lassen sich jedoch auch hier die Folgereaktionen der Additionsprodukte stark beeinflussen, wie das Beispiel der Nitrierung von 1,4-Dimethylnaphthalin zeigt: Während bei der Solvolyse mit Eisessig aus dem Addukt 20 unter $\mathrm{HNO}_{2}$-Abspaltung und Umlagerung ausschließlich das Naphtholacetat 21 entsteht, wird mit einem Gemisch aus Salpetersäure und Essigsäureanhydrid zunächst Essigsäure abgespalten. Die anschließende Addition von $\mathrm{NO}_{2}{ }^{\oplus}$ an die olefinische $\mathrm{CH}_{2}$-Gruppe und die Abspaltung von $\mathrm{NO}_{2}{ }^{\oplus}$ führen unter $\mathrm{Re}$ aromatisierung nur zum 1-Nitromethyl-4methyl-naphthalin.

Auch bei der Chlorierung von Alkylaromaten unter Ausschluß von Licht und Katalysatoren beobachtet man zum Teil Seitenket-<smiles>Cc1ccc(C)c2ccccc12</smiles>

$$
\text { 1,4-Dinethyl- }
$$

naphthalin

$-40^{\circ} \mathrm{C} \downarrow \mathrm{HNO}_{3} /\left(\mathrm{CH}_{3} \mathrm{CO}\right)_{2} \mathrm{O}$

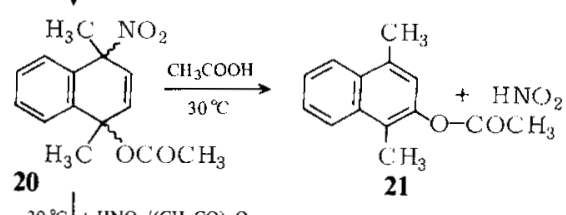

$30{ }^{\circ} \mathrm{C}$<smiles>C=C1C=CC(C)([N+](=O)[O-])c2ccccc21</smiles>

$+\mathrm{CH}_{3} \mathrm{COOH}$

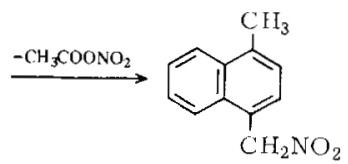

1 -Nitromethyl -

4 -methylnaphthalin

tensubstitution [11]. So führt die Chlorierung von Isodurol in Eisessig zwar noch überwiegend zu Kernsubstitution, daneben reagiert jedoch auch die Seitenkette, was sich über einen ipso-Angriff des Chlorelektrophils deuten läßt.

Für die Seitenkettenchlorierung von Polymethylbenzolen unter den oben angegebenen Bedingungen wurden auch Radikalkationen als Zwischenstufen vorgeschlagen [12]. Die

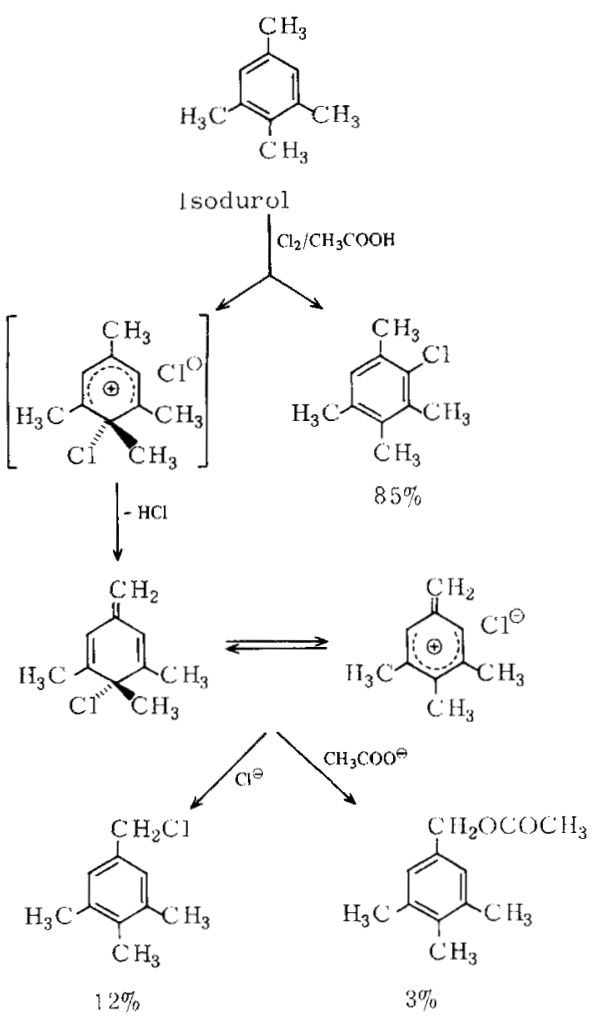

Bildung von Radikalkationen bei der Einwirkung von Elektrophilen auf Aromaten ist ein grundlegendes und wichtiges Problem bei vielen elektrophilen Aromatensubstitutionen.

\section{Radikalkationen als Zwischenstufen bei elektrophilen Aromatensubstitutionen}

Bei der Nitrierung von Phenolen oder Aminobenzolen, aber auch bei vielen anderen elektrophilen Aromatensubstitutionen wurden unkontrollierte Oxidationsreaktionen schon immer als störende Nebenreaktionen beobachtet. Mit der systematischen Bearbeitung oder gar präparativen Nutzung dieser nur schwer zu beherrschenden Reaktionen wurde allerdings erst begonnen. Wenn man die Oxidation im engeren Sinne als eine Einelektronenübertragung definiert und den Angriff eines Elektrophils als einen Zweielektronenübergang, dann sollte man eine Konkurrenz zwischen beiden Reaktionstypen erwarten, wenn das elektrophile Potential des Agens niedrig, sein Oxidationspotential für den Elektronenübergang jedoch ausreichend hoch ist oder wenn der elektrophile Angriff am Aromaten zum Beispiel aus sterischen Gründen erschwert ist. Charakteristische Beispiele für die Reaktion eines schwachen Elektrophils und für eine Reak tion unter sterischer Hinderung werden unten beschrieben. 
Von Radikalkationen als Zwischenstufen aus sind vielfältigere Folgereaktionen möglich, als von $\sigma$-Komplexen aus. Es entstehen also auch mehr Produkte. In Abbildung 9 sind die vier prinzipiell möglichen Folgereaktionen des Toluol-Radikalkations zusammengestellt.

Für die präparative Anwendung ist man bestrebt, einen oder mehrere Reaktionswege auszuschalten. So kann man beispielsweise Radikalkationen auch auf andere Weise erzeugen (elektrochemisch oder mit einem Oxidationsmittel) und damit z.B. Folgereaktionen mit E; also Weg a, ausschließen.

\section{Umsetzung von Mesitylen mit Perbenzoesäure}

Die Umsetzung der nur schwach elektrophilen Perbenzoesäure, $\mathrm{C}_{6} \mathrm{H}_{5}-\mathrm{CO}_{3} \mathrm{H}$, mit Mesitylen (Abbildung 10) führt selbst unter ausgeprägten Friedel-Crafts-Bedingungen zu einem Produktgemisch. Davon entsteht Mesitol wahrscheinlich als einziges Produkt durch elektrophile Hydroxylierung. Die Bildung der anderen Verbindungen läßt sich zwanglos mit Hilfe des Reaktionsschemas in Abbildung 9 über einen RadikalkationenMechanismus deuten. Das Chlorid-Ion für die Bildung von Chlormesitylen entstammt dem Trichlorhydroxyaluminat $\left(\mathrm{AlCl}_{3} \mathrm{OH}^{\ominus} \rightleftarrows\right.$ $\left.\mathrm{AlCl}_{2} \mathrm{OH}+\mathrm{Cl}^{\ominus}\right)$. Die Bildung des $\mathrm{Di}-$ phenylderivats 22 ist durch Dimerisierung (Weg b) denkbar; wegen des großen Mesitylen-Uberschusses ist jedoch auch ein Angriff von Mesitylen als Nucleophil am Radikalkation (Weg d) möglich. Die Reaktion zum Diphenylmethanderivat 23 verläuft über das Benzylkation (Weg c), das mit dem im Úberschuß vorhandenen Nucleophil Mesitylen zu 23 reagiert.

\section{Bromierung von $\mathrm{p}-\mathrm{Brom}-\mathrm{N}, \mathrm{N}$-dialkyl- anilinen}

Die Bromierung von N,N-Dialkylanilinen führt in Eisessig bei Raumtemperatur zu den 2,4-Dibromderivaten. Die erste Bromierung läuft in Chloroform bei $-40^{\circ} \mathrm{C}$ in para-Stellung ohne Schwierigkeiten ab, die Reaktion in der sterisch stark behinderten ortho-Position bleibt jedoch aus; stattdessen entstehen an der Luft zersetzliche 1:1-Additionsverbindungen ( $\pi$-Komplexe) mit Brom, z.B. 24 bei der Umsetzung von p-Brom-N,N-dimethylanilin. In Lösung gehen diese Addukte Folgereaktionen ein, die nur über Radikalkationen als Reaktionszwischenstufen erklärt werden können.

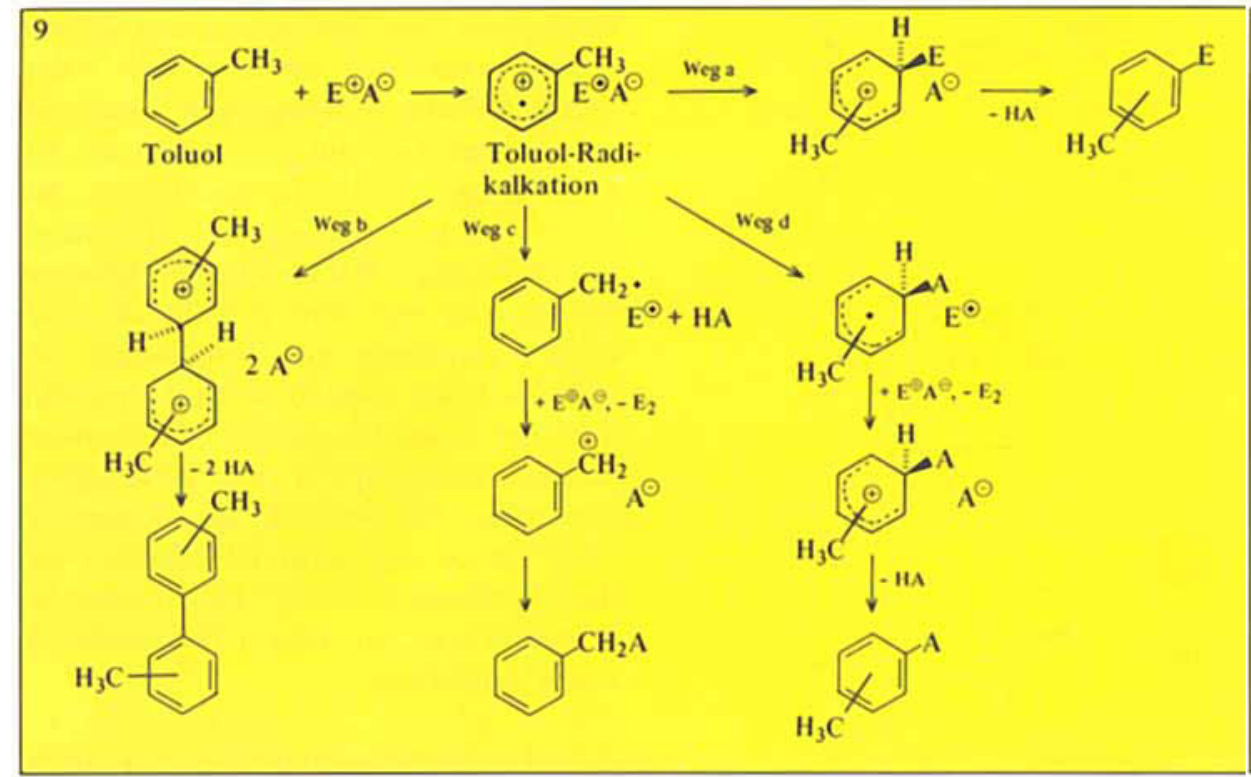

Beim Erwärmen des Adduktes 24 in Dimethylformamid (DMF) auf $20^{\circ} \mathrm{C}$ kommt es zu einem Einelektronenübergang unter Bildung des Radikalkations 25, das dann über Weg a, b oder c (s. Abbildung 9) abreagiert.

In Dimethylformamid entsteht über Weg a das Substitutionsprodukt o,p-

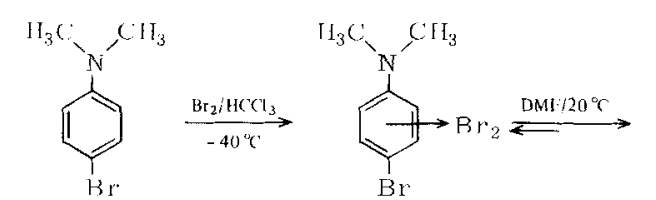

p-Brom -

$N, N$-dime

thylanilin

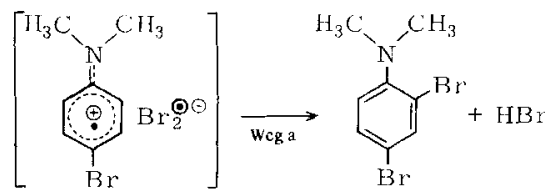

25

$\downarrow$ Weg c

$\stackrel{\mathrm{Hr}}{\stackrel{\mathrm{H}_{2} \mathrm{O} / \mathrm{H}^{\Phi}}{\rightarrow}}$

28

p-Brom -

$\mathrm{N}$-methylanilin

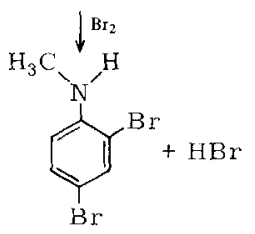

o,p-Dibrom $\mathrm{N}$-methylanilin

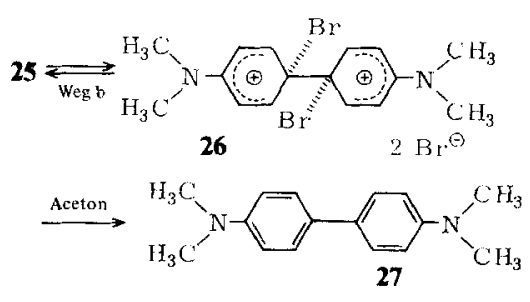

$+\mathrm{CH}_{3}-\mathrm{COCH} \mathrm{H}_{2} \mathrm{Br}+\mathrm{HBr}$

Dibrom-N,N-dimethylanilin und über Weg $c$ das entmethylierte o,p-Dibrom- $\mathrm{N}$-methylanilin. Eine Biphenylbildung über die Dime risierung des Radikalkations 25 (Weg b) wird bei der Umsetzung in Dimethylformamid nicht beobachtet, da offensichtlich die Abspaltung von $\mathrm{Br}^{\oplus}$ aus 26 zu 27 unter diesen Bedingungen nicht möglich ist. Stattdessen zerfällt $\mathbf{2 6}$ wieder in zwei Radikalkationen 25, die auf den beiden vorher beschriebenen Wegen abreagieren. Durch Zugabe von Reduktionsmitteln, z.B. Natriumsulfit, oder durch Umsetzung des $\pi$-Komplexes 24 in Aceton - das in der Lage ist, $\mathrm{Br}^{\oplus}$ aufzunehmen - kann die Reaktion von 25 überwiegend über Weg b gelenkt werden; neben kleineren Anteilen o,p-Dibrom-N,N-dimethylanilin und $o, p-D i b r o m-N$-methylanilin entsteht unter diesen Bedingungen zu über $60 \%$ das Benzidin 27. Das Verhältnis der Produkte, die über Weg a oder Weg c entstehen, hängt wie zu erwarten von den Alkylsubstituenten am Stickstoff ab. So wird das N-Isopropyl$\mathrm{N}$-methylanilin-Addukt in Dimethylformamid eher entalkyliert als am Kern substituiert. Weg a ist hier aus sterischen Gründen wegen des größeren Alkylrestes erschwert, während Weg c aufgrund der besseren Stabilisierung der Zwischenverbindungen ( $z$. B. vom Typ 28) begünstigt wird. Es muß fest- 


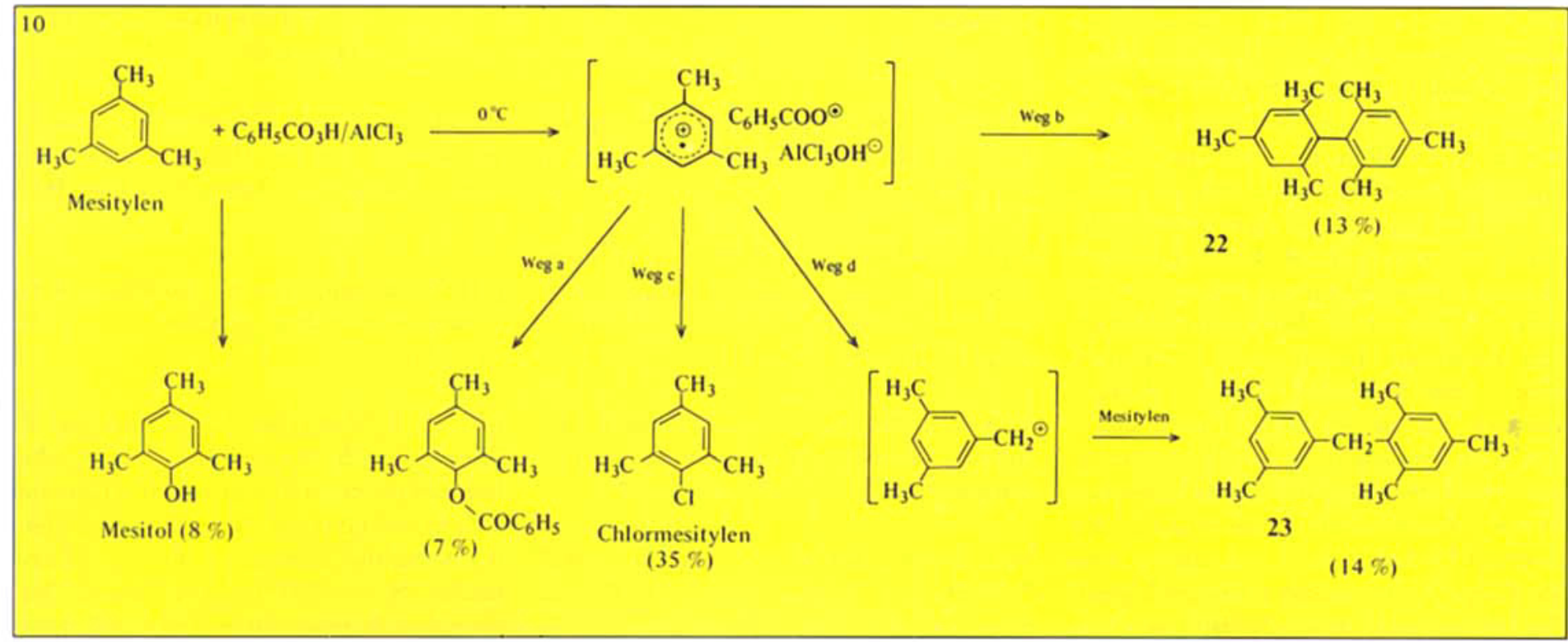

Abb. 9. Reaktionsmöglichkeiten von Radikalkationen. Weg a: Addition des Radikals $\mathrm{E}^{\cdot}$ zum $\sigma$-Komplex und Deprotonierung zum Substitutionsprodukt. Weg b: Dimerisierung über eine dikationische Zwischenstufe und Rearomatisierung zu einem Biphenyl. Weg c: Protonenabspaltung aus der Methylgruppe, Weiteroxidation zum Benzylkation und Addition des Anions. Weg d: Addition des Anions zu einem Radikal- $\sigma$-Komplex, Weiteroxidation zum kationischen $\sigma$-Komplex und anschließende Deprotonierung zum Substitutionsprodukt.

Abb. 10. Radikalkationen als Zwischenstufen bei der "elektrophilen Hydroxylierung“ von Mesitylen. gehalten werden, daß o,p-Dibrom-N,N-dimethylanilin nicht nur auf dem oben beschriebenen Weg a, sondern auch über eine normale elektrophile Substitution entstehen kann.

Radikalkationen als Zwischenstufen bei elektrophilen Aromatensubstitutionen sind grundsätzlich immer dann in Erwägung zu ziehen, wenn Biphenyle entstehen, da deren Bildung auf andere Weise kaum zu erklären ist. Das wird z.B. bei vielen Nitrierungen beobachtet.

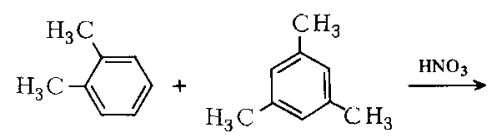

o-Xylol Mesitylen

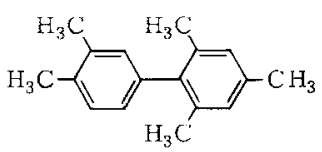

+ Substitutionsprodukte

Sind $\pi$-Komplexe Zwischenstufen bei elektrophilen Aromatensubstitutionen?

$\pi$-Komplexe werden häufig als $Z$ wischenstufen bei elektrophilen Aromatensubstitutionen postuliert. Ihre Bildung wird als geschwindigkeitsbestimmend angenommen, wenn bei der Zweitsubstitution Abweichungen von den üblichen - durch die $\sigma$ Komplexstabilitäten bedingten - Isomerenverteilungen auftreten.

Da die $\pi$-Komplexe auf der Reaktionsko- ordinate vor den entsprechenden $\sigma$-Komplexen liegen, werden die oben beschriebenen Befunde besonders bei Reaktionen mit frühem Ubergangszustand zu erwarten sein und - nach dem Hammond-Postulat [13] - bei sehr energiereichen Reaktanden.

Gerade bei Nitrierungen treten die erwähnten Abweichungen auf; das hängt damit zusammen, daß das bei allen Nitrierungen auftretende Nitryl-Kation, $\mathrm{NO}_{2}^{\oplus}$, zu den energiereichen Elektrophilen gehört. Da es keinen überzeugenden Beweis für $\pi$-Komplexe als notwendige $Z$ wischenstufen bei elektrophilen Aromatensubstitutionen gibt, kann das abweichende Verhalten bei Nitrierungen auch über einen Radikalkationen-Mechanismus gedeutet werden, mit dem alle experimentellen Befunde übereinstimmen [14]. Allgemein kann man bei den Umsetzungen von Elektrophilen mit Aromaten immer dann Radikalkationen erwarten, wenn die Elektronenübertragung von Aromaten zum Elektrophil exotherm verläuft.

Wenn auch in dervorliegenden Zusammenfassung die mechanistischen Gesichtspunkte der neuen Entwicklungen auf dem Gebiet der elektrophilen Aromatensubstitution stärker im Vordergrund stehen, so ist doch bei vielen Beispielen auch die synthetische Bedeutung dieser Entwicklungen zu erkennen. Wegen der in den letzten Jahren völlig veränderten Rohstoffsituation und der ständig steigenden ökologischen Anforderungen an chemische Prozesse sind bei vielen heute noch in der Industrie angewandten Aromatenreaktionen für die absehbare Zukunft Probleme vorauszusehen. Das gilt besonders 
für Verfahren, bei denen unerwünschte Isomere entstehen, die vernichtet werden müssen, und für Verfahren, bei denen große Mengen konzentrierter Säuren (z.B. Nitrierungen oder Sulfonierungen) oder Laugen ( $\mathrm{KOH}-S c h m e l z e$ von Sulfonsäuren) verwendet werden müssen. Die Becinflussung der Isomerenverteilung und ökologisch günstigcre Reaktionsverfahren könnten diese Probleme lösen helfen. Die Isomerenverteilungen sind durch die Lenkung der Reaktionen über ipso-Additionen oder Radikalkationen zu beeinflussen. Durch Addition von Nucleophilen an RadikalkationZwischenstufen lassen sich Substituenten einführen (z.B. $\mathrm{OH}, \mathrm{NH}_{2}, \mathrm{~F}$ ), die durch direkte elektrophile Substitution nur schwer oder überhaupt nicht eingeführt werden können. Die Erforschung dieser vielfältigen neuen Möglichkeiten bei der elektrophilen Aromatensubstitution steht allerdings, was ihre praktischen Anwendungen betrifft, noch am Anfang.

\section{Literatur}

[1] G. A. Olah: "Mechanism of electrophilic aromatic substitutions". Acc. Chem. Res. 4, 240 (1971).

[2] F. Effenberger und P. Menzel: "б-Komplexe bei der Halogenierung von Aminobenzolen - Isolierung und Folgereaktionen". Angew. Chem. 84, 954 (1972).

[3] S. R. Hartshorn: "Non-conventional electrophilic aromatic substitution and related reactions". Chem. Soc. Rev. 1974, 167.

[4] J. March: "Advanced Organic Chemistry". McGraw Hill Comp., New York 1977, 2. Aufl., S. 472.

[5] F. Effenberger und P. Menzel: „Isomerisierung, Enthalogenierung und Entalkylierung von Brom-N, N-dialkylanilinen mit Mineralsäuren“. Chem. Ber. 110, 1342 (1977).

[6] M. Tashiro und G. Fukata: "Selective electrophilic aromatic substitutions via positional protective groups. A revicw". Org. Prep. Proc. Int. 8, 51 (1976).

[7] D. E. Pearson and C. A. Buehler: "Unusual electrophilic aromatic substitution in synthesis“. Synthesis 1971, 455.

[8] F. Effenberger, H. Klenk und P. L. Reiter: „Neue Aspekte zur Fries-Umlagerung". Angew. Chem. 85, 819 (1973).

[9] R. B. Moodie und K. Schofield: "Ipsoattack in aromatic nitration". Acc. Chem. Res. 9, 287 (1976); P.B.D. de la Mare: "Pathways in Electrophilic Aromatic Substitutions. Cyclohexadienes and Related Compounds as Intermediates in Halogenation". Acc. Chem. Res. 7, 361 (1974).

[10] H. Suzuki: "Side-reactions in aromatic nitration; some synthetic aspects". Synthesis $1977,217$.

[11] G. Illuminati, L. Mandolini und A. Patara: "Non-conventional paths in electrophilic aromatic reactions - XI. The orientation in the chlorination of isodurene in acetic acid“. Tetrahedron Lett. 1972, 4161; E. Baciocchi, L. Mandolini und A. Patara: "Non-conventional paths in electrophilic aromatic reactions - XIV. Intervention of ion pairs in the mechanism of the side-chain chlorination of polyalkylbenzenes". Tetrahedron Lett. 1975, 2268.

[12] J. K. Kochi: "Electrophilic aromatic substitution: Electron transfer routes in side chain substitutions“. Tctrahedron L.ett. 1975, 41.

[13] D. Farrcasiu: "The use and misuse of the Hammond postulate“. J. Chem. Educat. 52, 76 (1975).

[14] E. B. Pedersen, T. E. Peterden, K. Torssell und S.-O. Lawesson: Relationship between the properties of radical cations and the rate constants and the substitution pattern in electrophilic aromatic substitution". Tetrahedron 29, 579 (1973); Ch. L. Perrin: "Necessity of electron transfer and a radical pair in the nitration of reactive aromatics". J. Am. Chem. Soc. 99, 5516 (1977).

Prof. Dr. F. Effenberger, geb. 1930 in Goldenstein, Nordmähren. Chemiestudium an der Universität Stuttgart. 1959 Promotion über ein Problem aus der Heterocyclenchemie. 1964 Habilitation, 1965 Forschungsaufenthalt an der University of Michigan (Ann Arbor), 1977 Gastprofessor an der Cornell University (Ithaca, NY). Seit 1972 als Nachfolger von $\mathrm{H}$. Bredereck Professor für Organische Chemie an der Universität Stuttgart. Arbeitgebiete: Aktivierte Olefine und Aromaten (Enolether, Aminobenzole), neue elcktrophile Agentien Austrittsgruppenprobleme, Radikalkationen als Reaktionszwischenstufen, Synthese und Chemie von Aminosäuren. 